\title{
Seasonal Adjustment Algorithm
}

\author{
Kirill Spiridonov \\ Saratov State University, Saratov, Russian Federation, \\ kir.spiridonov@gmail.com
}

\begin{abstract}
The paper presents an algorithm for smoothing time series with seasonality. The proposed method is based on median smoothing and the Hodrick-Prescott decomposition. Using a software implementation in the $R$ language, the correctness of the developed algorithm is checked; it is also compared with other seasonal smoothing methods.
\end{abstract}

Index Terms-Seasonal adjustment, Median adjustment, Hodrick-Prescott Filter

\section{INTRODUCTION}

The dynamics of some economic processes in the same time periods in different years may be similar, that is, have seasonality. The presence of seasonality in the time series can complicate the analysis and even lead to incorrect conclusions. And when studying the dependencies between economic processes, the presence of a similar seasonal component in several completely unrelated series may lead to the erroneous conclusion that there is a stable, statistically significant relationship between these processes. Thus, the task of eliminating seasonality is very important in the analysis of time series.

In the general case, the time series $y_{t}$ consists of the trendcycle $-t c_{t}$, seasonal $-s_{t}$ and irregular $-i_{t}$ components. Some authors (for example, [1] and [2]) separate the trend-cycle component that corresponds to the potential level of a certain economic indicator and the cyclical component associated with fluctuations in the economic situation. However, in the present work, the difference between the trend and the trend-cyclic component is bypassed. In addition, most modern seasonal smoothing algorithms also do not allocate the cycle into a separate component.

By the method of component aggregation, time series are additive

$$
y_{t}=T R_{t}+S_{t}+I_{t}
$$

and multiplicative

$$
y_{t}=T R_{t} \cdot S_{t} \cdot I_{t}
$$

Most economic processes have a multiplicative structure. The use of the additive model is advisable in the case when as the level of the series increases, the amplitude of seasonal fluctuations is constant.

The exponential smoothing, proposed by Brown [3], has been one of the first methods for smoothing time series. This method is applicable for series fluctuating a round a certain constant level, without trends and seasonal components. However, the presence of a structure in time series in some way changes the state of affairs. For example, for series with a linear trend, Brown showed [4] that exponential smoothing does give not satisfactory results.

To smooth time series with a linear trend, the Brown method [5] and the Holt method [6] have proven themselves well. The described methods aren't suitable for smoothing time series with seasonality. Winters proposed a modification of the Holt method [7], taking into account the presence of a multiplicative seasonal component in the series. For the additive factor, there is another modification of the Holt method, called the TheilWedge method [8].

The most modern time series smoothing algorithms also take into account the structure. This algorithms are based on the decomposition of the initial series into a trend, seasonal and irregular components. Thus, the smoothed series is a combination of a trend and a random component. The most popular seasonal adjustment algorithms are the nonparametric X-series algorithms ([9]-[11]) and the parametric TRAMO/SEATS method [12].

\section{Seasonal Adjustment Algorithm}

Before the description of the seasonal adjustment procedure, we note that the algorithm involves working only with monthly and quarterly data.

Suppose that we have a time series $-x_{1}, \ldots x_{n}$, then the number of full years for which there are observations can be defined as

$$
T=\left[\frac{n}{p}\right]+\gamma, \quad p=4,12,
$$

where $[x]$ - the integer part of a number $x$, and

$$
\gamma=\left\{\begin{array}{lll}
0, & n & \bmod p=0 \\
1, & n & \bmod p=1
\end{array}\right.
$$

\section{A. Data Transformation}

Depending on the aggregation of the main components of the time series, the series can have an additive or multiplicative structure. Working with the additive model seems more preferable because the seasonal wave becomes more uniform and does not evolve over time.

Let $x_{1}, \ldots, x_{n}$ be the time series with a multiplicative structure, then the transition to the additive model $y_{1}, \ldots, y_{n}$ is carried out

$$
\begin{gathered}
y_{t}=\ln \left(x_{t}+|\alpha|\right), \text { where } \\
\alpha=\left\{\begin{array}{l}
\min _{t}\left(x_{t}\right)-1, \text { if } \quad \exists t: x_{t} \leq 0 ; \\
0 .
\end{array}\right.
\end{gathered}
$$




\section{Estimate of Seasonal Component}

Thus, at the first step, data linearization is carried out in order to move to an additive model

$$
y_{t}=C_{t}+S_{t}+I_{t}
$$

It is also worth noting that if the series has an additive structure, then data conversion is not required.

\section{B. Hodrick-Prescott Filter}

The Hodrick-Prescott filter is used to remove the cyclical component of a time series. This method was first used by Hodrick and Prescott to analyze post-war US business cycles [13].

A filter is a solution to an optimization problem. On the one hand, the smoothed series should not differ much from the original series, that is, it is necessary to minimize the sum of the squared deviations.

$$
\sum_{t=1}^{n}\left(y_{t}-L_{t}\right)^{2} \longrightarrow \min
$$

On the other hand, the series should be smooth enough and change not too sharply

$$
\sum_{t=2}^{n-1}\left(\left(L_{t+1}-L_{t}\right)-\left(L_{t}-L_{t-1}\right)\right)^{2} \longrightarrow \min
$$

Thus, the filter is a two-sided linear filter that calculates the smoothed series $L_{t}$ of the time series $y_{t}$ by minimizing the difference of the series $L_{t}$ and $y_{t}$, and minimizing the sum of the second differentiated series $L_{t}$

$\sum_{t=1}^{n}\left(y_{t}-L_{t}\right)^{2}+\lambda \sum_{t=2}^{n-1}\left(\left(L_{t+1}-L_{t}\right)-\left(L_{t}-L_{t-1}\right)\right)^{2} \longrightarrow$ min.

The parameter $\lambda \geq 0$ is a measure of the smoothness of the resulting series. For $\lambda=0$, the smoothed series exactly matches the original one. If $\lambda \longrightarrow+\infty$, then the series approaches a linear trend. In the literature, there are different approaches to the definition of $\lambda$ (for example, [14]), however, the following values are considered a "classic" variant [15]

- $\lambda=100$, for annual data;

- $\lambda=1600$, for quarterly data;

- $\lambda=14400$, for monthly data.

As an alternative, the Baxter-King filter was considered. However, using this method, the observations at the ends of the sample fall out of consideration. Therefore, the results of this filter are not presented in the work. The asymmetric Christiano-Fitzgerald filter does not have this drawback, but an additional step is required before its application - checking the series for the presence of unit roots, since the filter works differently on stationary and integrated series.

Thus, the use of the Hodrick-Preccott filter is related to its simplicity (in fact, it is only necessary to set the parameter $\lambda$ ) and the lack of the need for preliminary work with the series.
Let's apply the Hodrick-Prescott filter to $y_{t}$. Now we get the first estimate of the trend $L_{t}^{1}$ and subtracting it from the original series, we get the sum of the seasonal and irregular components

$$
S_{t}+I_{t}=y_{t}-L_{t}^{1}
$$

Now it is needed to separate the irregular component from the seasonal wave. For this, it is necessary to group seasonal components from $S_{t}+I_{t}$ by seasonality periods. We have $p$ distributions of seasonal coefficients $s_{i}^{1}, \ldots s_{i}^{T}$, where $i \in$ $[1, p]$. Meanwhile, in some sets the coefficient of the last period $T$ may be absent.

Each of the series $s_{i}^{1}, \ldots s_{i}^{T}$ is smoothed by a moving median, with a window width of three. In many algorithms, the first estimate of seasonality is obtained using a moving (weighted) average. Compared with the average, the median estimate of the distribution center is more robust, which allows us to more effectively exclude outliers from consideration. Thus, at this stage, we have the median smoothed coefficients $S_{t}^{1}$.

A moving window with a width of three observations is used for several reasons. Firstly, such a window width filters out errors well, while the series does not become too smooth (the same window width is used, for example, in some X-series algorithms). Secondly, already for relatively short time series (from 4 years) you can get a quality estimate of seasonality.

Since this approximation of the seasonal wave is rather rough, it must be supplemented with errors limited to a certain value. In addition, in the absence of outbursts inside the window, the smoothed curve will have a "jagged" look, so it needs to be modified. To do this, we form a series of errors

$$
e_{t}=S_{t}+I_{t}-S_{t}^{1}
$$

and calculate the standard deviation $\sigma$ for it. We compose a new series of errors from such members $e_{t}$ that do not exceed $2 \sigma$, and instead of those that exceed, we use $2 \sigma$ :

$$
\widehat{e}_{t}= \begin{cases}e_{t}, & e_{t} \leq 2 \sigma \\ 2 \sigma, & e_{t}>2 \sigma\end{cases}
$$

Let us add the resulting series $\widehat{e}_{t}$ to the median-smoothed series $S_{t}^{1}$ and smooth it with a third-order moving average in three different ways:

1) The window consists of the current and 2 previous observations;

2) The window consists of the current and 2 subsequent observations;

3) The window consists of the current, previous and subsequent observations.

Thus, we get three smoothed series $S_{t}^{21}, S_{t}^{22}$ and $S_{t}^{23}$. As the final estimate of the seasonal wave, we use the average value of the obtained series, i.e.

$$
S_{t}^{2}=\frac{S_{t}^{21}+S_{t}^{22}+S_{t}^{23}}{3} .
$$


Further, we will no longer work with each individual seasonal period, but we will work with a seasonal wave within each year under consideration. To filter out the remaining seasonal errors, we are smoothing out the seasonal components of each year with a third-order moving average in three different ways, as described above. As a result, we obtain three smoothed series $\left(S_{t}^{31}, S_{t}^{32}\right.$ and $S_{t}^{33}$ ), and use the average as the final estimate of the seasonal wave value from obtained series.

$$
S_{t}^{3}=\frac{S_{t}^{31}+S_{t}^{32}+S_{t}^{33}}{3} .
$$

Note that this procedure is carried out only for series with a monthly frequency. For quarterly data, this step is omitted, because otherwise the final series would be too smooth, and the seasonal estimate would be distorted.

Now it remains to normalize the seasonal component. In the case of the additive model, the sum of seasonal coefficients during the year is 0 , and in the case of the multiplicative model, their product is 1 :

- for the additive model from each coefficient within one year, subtract their average value;

- for the multiplicative model, we divide each coefficient within one year by their geometric mean.

Thus, we have the normalized seasonal coefficients $\widetilde{S}_{t}^{3}$, and a smoothed series $\left(y_{t}-\widetilde{S}_{t}^{3}\right)$. The final trend estimate can be obtained by applying a Hodrick-Prescott filter to the smoothed series.

\section{PRACTICAL IMPLEMENTATION}

We use $\mathrm{R}$ language for the implementation of the seasonal adjustment algorithm. The choice of this software product is due to several factors. Firstly, at the moment, $\mathrm{R}$ is one of the most popular and most powerful languages for statistical calculations and modeling, while its syntax is quite simple. Secondly, due to its high popularity, there are a large number of user libraries that implement additional functionality and allow you to solve almost any task in a few simple steps.

To develop the seasonal smoothing script, we used both standard functions and procedures of the R language [16], as well as functions developed by third-party users. In particular, the functions included in the freeware packages mFilter [17] and psych [18] were used.

Several series of macroeconomic indicators were used to compare the smoothing algorithms. The information base of the study is the resources of the Federal State Statistics Service. Several series were smoothed out: retail turnover of non-food products, consumer price index for sugar, unemployment rate, volume of investments in fixed assets. The indicators were selected in such a way as to check all the options for the algorithm to work (on monthly and quarterly data, with and without logarithmic transformation).

The TRAMO/SEATS and X-12-ARIMA algorithms were chosen as alternative methods of seasonal smoothing to compare and verify the correctness of the developed algorithm. The choice of these methods is due to their popularity: both algorithms are integrated into most modern econometric
After that, the new weights are normalized again, as in the first part of (2). Finally, using the new weights, all seasonal coefficients are recalculated using (1). 


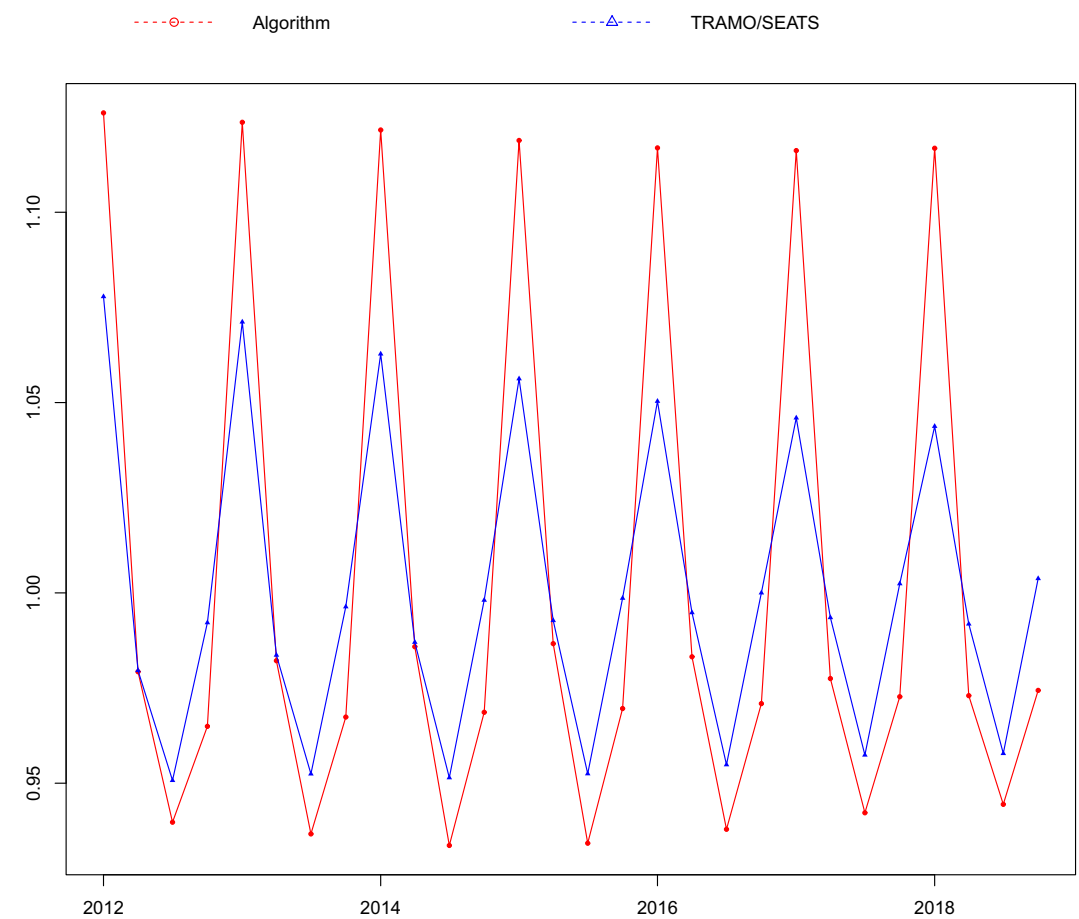

Fig. 1. Seasonal component: unemployment rate.

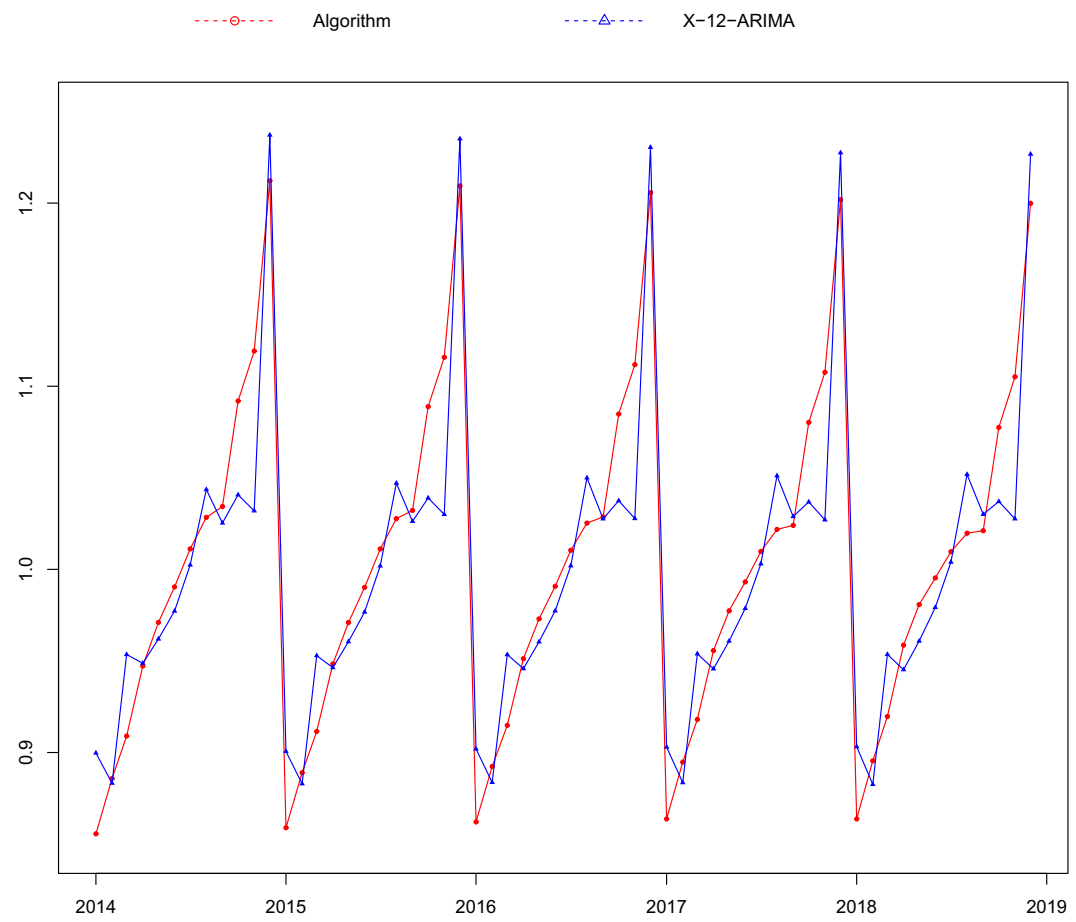

Fig. 2. Seasonal component: retail turnover.

packages and are used by many organizations for the seasonal smoothing procedure.

The results of the comparison of the algorithms are shown in the Fig. 1 and 2. In general, it can be noted:
- the seasonal component obtained using the developed algorithm is similar to the seasonality identified by TRAMO/SEATS and X-12-ARIMA: the peaks of the seasonal wave fall on the same periods; trends observed 


\section{REFERENCES}

over one year are similar; the amplitude of the oscillations and some evolution of the wave in time are also consistent;

- the difference lies in the uniformity of the seasonal wave. In almost all cases, TRAMO/SEATS shows a more uniform result, which is due to the principle of its construction: when smoothing any series, the algorithm tries to minimize the evolution of seasonality over time. In cases where seasonality has evolved, the result of the developed algorithm is closer to X12-ARIMA.

On the one hand, the stability of the seasonal factor, like that of TRAMO/ SEATS, looks attractive, but on the other hand, the evolution of economic processes under the influence of many factors is ongoing (including the evolution of the seasonal component) and in this case the requirement for the stability of the seasonal component is quite strict. The result of the X-12-ARIMA in some cases, on the contrary, allows for too much seasonal variation. The developed algorithm in the sense of seasonal factor stability is located between these two smoothing methods and can be used for working with data.

Another advantage of the developed algorithm is that in the process of smoothing median smoothing is used, which allows to get rid of outliers and random errors that may be present in the data. This eliminates the need to separately model outliers (for example, using dummy variables) and generally simplifies series processing, since in some cases the quality of seasonal smoothing of the TRAMO/SEATS and X-12-ARIMA algorithms significantly depends on the selected outliers.

\section{ACKNOWLEDGMENTS}

This research was funded by Russian Science Foundation, grant number 19-18-00199.
[1] Lukashin, Y.P., Adaptive methods for time series short term forecasting. Finance and Statistics, 2003.

[2] Nosko, V.P., Econometrics. Book 1. Part 1 and 2. Delo, 2011.

[3] R. Brown, Exponential Smoothing for Predicting Demand. Little, 1956.

[4] C. Lewis, Industrial and Business Forecasting Methods: A Practical Guide to Exponential Smoothing and Curve Fitting, ser. Butterworth scientific. Butterworths Scientific, 1982.

[5] R. Brown, Smoothing, forecasting and prediction of discrete time series, ser. Prentice-Hall international series in management. Prentice-Hall, 1963.

[6] C. Holt, "Forecasting trends and seasonal by exponentially weighted averages," Office of Naval Research Memorandum, vol. 20, 011957.

[7] P. R. Winters, "Forecasting sales by exponentially weighted moving averages," Management Science, vol. 6, pp. 324-342, 041960.

[8] H. Theil and S. Wage, "Some observations on adaptive forecasting," Management Science, vol. 10, pp. 198-206, 011964.

[9] J. Shiskin, A. H Young, and J. C Musgrave, "The x-11 variant of the census method ii seasonal adjustment program," 011967.

[10] X-12-ARIMA Reference Manual, U.S. Census Bureau, Washington, DC, 2011.

[11] X-13-ARIMA-SEATS Reference Manual, U.S. Census Bureau, Washington, DC, 2015.

[12] V. G'omez and A. Maravall, "Programs TRAMO and SEATS: instructions for the user," Mimeo, Banco de España, 011997.

[13] R. Hodrick and E. Prescott, "Post-war US business cycles: An empirical investigation," vol. 8, pp. 231-247, 011981.

[14] M. Ravn and H. Uhlig, "On adjusting the HP filter for the frequency of observations," The Review of Economics and Statistics, vol. 84, 02 1997.

[15] C. Favero, Applied Macroeconometrics. Oxford University Press, 2001.

[16] R. Kabacoff, $R$ in Action: Data Analysis and Graphics with $R$. Greenwich, CT, USA: Manning Publications Co., 2015

[17] M. Balcilar, mFilter: Miscellaneous time series filters, 2007, $\mathrm{r}$ package version 0.1-3. [Online]. Available: https://CRAN.Rproject.org $/$ package $=$ mFilter

[18] W. Revelle, psych: Procedures for Psychological, Psychometric, and Personality Research, Northwestern University, Evanston, Illinois, 2018, r package version 1.8.12. [Online]. Available: https://CRAN.Rproject.org/package $=$ psych 